\title{
Neurologic Mechanisms Underlying Voiding Dysfunction due to Prostatitis in a Rat Model of Nonbacterial Prostatic Inflammation
}

\author{
Jee Soo Park, Mei Hua Jin, Chang Hee Hong \\ Department of Urology, Yonsei University College of Medicine, Seoul, Korea
}

Purpose: The neurological molecular mechanisms underlying the voiding dysfunction associated with nonbacterial chronic prostatitis/chronic pelvic pain syndrome remain poorly understood. In this study, we assessed whether prostate inflammation activated bladder afferent neurons, leading to bladder dysfunction, and sought to elucidate the underlying mechanisms.

Methods: Thirty male Sprague-Dawley rats were divided into 3 groups: sham-saline, formalin-injected, and capsaicin-pretreated and formalin-injected. Chemical prostatitis was induced by $0.1 \mathrm{~mL}$ of $10 \%$ buffered formalin injected into the ventral prostate. Capsaicin was injected subcutaneously to desensitize capsaicin-sensitive nerves. In each group, conscious cystometry was performed, and c-fos expression within the spinal cord was determined immunocytochemically. Double immunofluorescent staining with c-fos and choline acetyltransferase (ChAT) was performed. On the third day after pseudorabies virus (PRV) infection, c-fos and PRV double-staining was performed.

Results: Intraprostatic formalin significantly increased the maximal voiding pressure and decreased the intercontraction interval, compared with controls. Pretreatment with capsaicin significantly reversed these effects. More c-fos-positive cells were observed in the sacral parasympathetic nucleus (SPN) and dorsal gray commissure (DCM) in the prostatitis group than in the sham group. c-fos-positive cells decreased in the capsaicin-pretreated group. Preganglionic neurons labeled by c-fos and ChAT were observed in the SPN in rats with prostatitis. Interneurons labeled by c-fos and PRV were identified in the DCM after PRV infection.

Conclusions: Our results suggest that prostate inflammation activates afferent nerve fibers projecting to the lumbosacral spinal cord, producing reflex activation of spinal neurons innervating the bladder and bladder hyperreflexia. This is mediated by capsaicin-sensitive prostate afferent neurons.

Keywords: Prostatitis; Voiding dysfunction; Prostate afferent

- Fund/Grant Support: This study was supported by a faculty research grant of Yonsei University College of Medicine (No. 6-2007-0160).

- Research Ethics: All experiments were performed in accordance with institutional guidelines and approved by the Yonsei Animal Research Ethics Committee.

- Conflict of Interest: No potential conflict of interest relevant to this article was reported.

\section{- HIGHLIGHTS}

- Our study presents a detailed characterization of the neurological mechanisms of voiding dysfunction in rats with nonbacterial prostatic inflammation.

- A novel aspect of this study is that it presents results from the analysis of efferent neurons, as well as afferent and spinal neurons.

- These findings may provide a useful and clinically relevant framework for approaching the treatment of chronic prostatitis/chronic pelvic pain syndrome.

Corresponding author: Chang Hee Hong (iD https://orcid.org/0000-0002-0946-7702 Department of Urology, Gangnam Severance Hospital, Yonsei University College of Medicine, 211 Eonju-ro, Gangnam-gu, Seoul 06273, Korea

E-mail: chhong52@yuhs.ac / Tel: +82-2-2019-3473 / Fax: +82-2-3462-8887

Submitted: June 8, 2018 / Accepted after revision: June 20, 2018 (c) (i) (2) This is an Open Access article distributed under the terms of the Crecommons.org/licenses/by-nc/4.0/) which permits unrestricted non-commercial use, distribution, and reproduction in any medium, provided the original work is properly cited. 


\section{INTRODUCTION}

Prostatitis is an increasingly common urologic problem, with a worldwide prevalence of $2 \%-10 \%$ in adult men [1]. According to the National Institutes of Health, patients with prostatitis are classified into 4 categories: category I (acute bacterial prostatitis), category II (chronic bacterial prostatitis), category III (chronic prostatitis/chronic pelvic pain syndrome [CP/CPPS]), and category IV (asymptomatic inflammatory prostatitis) [2]. The vast majority of diagnosed cases of prostatitis are in the form of CP/CPPS [3], which is characterized by pelvic pain and lower urinary tract symptoms [4]. However, the underlying mechanisms of the accompanying voiding dysfunction are not fully understood.

Recent evidence has suggested that capsaicin-sensitive C-fiber bladder afferents contribute to the development of overactive bladder and bladder pain in various other pathological conditions that are similar to $\mathrm{CP} / \mathrm{CPPS}$, such as spinal cord injury, bladder outlet obstruction, and interstitial cystitis [5-7]. The role of C-fiber afferents in CP/CPPS has been described in a clinical study [8], but definitive experimental evidence through animal studies has not yet been reported. Vera and Meyer-Siegler [9] noted increased proinflammatory cytokines in the bladder with prostatic inflammation, suggesting that a viscerovisceral reflex involving the lumbosacral spinal cord might be associated with voiding dysfunction in prostatitis. Using pseudorabies virus (PRV), Nadelhaft et al. $[10,11]$ demonstrated that the spinal neurons innervating the bladder and prostate were separate, but neurons from the bladder and prostate were mainly located in the dorsal gray commissure (DCM), suggesting that neurons originating from these 2 organs interact and coordinate through interneurons. Thus, it has been postulated that hyperexcitability of the bladder afferent pathway, followed by sensitization of C-fiber afferents, leads to voiding symptoms in patients with prostatitis.

In this study, we evaluated whether voiding dysfunction in prostatitis is caused by prostate inflammation-mediated activation of lumbosacral interneurons that innervate both the bladder and prostate by assessing PRV, choline acetyl transferase (ChAT), and c-fos expression. We also examined whether spinal neuron activation was mediated by capsaicin-sensitive prostate afferents, as capsaicin-sensitive bladder afferents are associated with bladder hyperactivity.

\section{MATERIALS AND METHODS}

\section{Animals and surgical procedures}

Male Sprague-Dawley rats $(n=30)$, weighing $235-310 \mathrm{~g}$, were randomly assigned to 1 of 3 different groups $(n=10)$ : sham rats injected with saline, rats with formalin-induced prostatic inflammation, and capsaicin-pretreated rats with formalin-induced prostatic inflammation. All experiments were performed in accordance with institutional guidelines and approved by the Yonsei Animal Research Ethics Committee.

Immediately after anesthesia with isoflurane, $0.1 \mathrm{~mL}$ of $10 \%$ buffered formalin was injected into the ventral prostate using a 30-gauge needle to induce prostatitis. Control rats received a similar injection with $0.1 \mathrm{~mL}$ of saline. To exclude formalin-induced bladder irritation, 3 rats were injected with formalin containing $100 \mathrm{mM}$ methylene blue; dye was not observed in the urine or bladder, indicating that the formalin did not spread to the bladder.

\section{Capsaicin pretreatment}

To determine whether the neurons activated by formalin were sensitive to capsaicin, capsaicin $(50 \mathrm{mg} / \mathrm{kg}$ ) was injected subcutaneously 5 days before prostatitis induction. To prevent capsaicin-mediated respiratory failure, atropine $(0.2 \mathrm{mg} / \mathrm{kg})$, terbutaline $(0.2 \mathrm{mg} / \mathrm{kg})$, and aminophylline $(20 \mathrm{mg} / \mathrm{kg})$ were injected intraperitoneally before the capsaicin. Capsaicin desensitization was evaluated by the eye wipe test before prostatitis induction.

\section{Tissue preparation and harvesting}

To determine whether activation of spinal neurons of the bladder was caused by prostatitis, we induced prostatitis in rats chemically. To trace the bladder spinal neurons, PRV was injected 3 days before prostatitis induction, and PRV expression was analyzed. c-fos expression was assessed to demonstrate spinal neuron activation by prostatitis induction. Cholinergic neurons were visualized by ChAT labeling to distinguish parasympathetic innervation arising from preganglionic neurons (PGNs), which cause detrusor muscle contractions.

\section{Detrusor muscle injection of PRV}

The urinary bladder was exposed by a midline abdominal incision, and PRV (Bartha strain, $2.6 \times 10^{8}$ plaque-forming units per milliliter, donated by Bong Hee Lee, Jeju National University, Jeju, Korea) was injected with a Hamilton micropipette. Bartha is an attenuated PRV strain that reliably labels neuronal pathways via 
transneuronal transport. Two injections $(2 \mu \mathrm{L}$ each) were made into the ventral wall of the urinary bladder. To prevent the virus from spreading to the prostate, injections were confined to the bladder body, far from the prostate, and the expelled fluids were carefully washed with saline to prevent spread to nearby tissues. The animals were sacrificed 3 days after the PRV injection, at which time the spinal cord was removed for cryostat sectioning.

\section{Spinal cord sectioning}

While anesthetized with ether, the animals were sacrificed 2 hours after saline or formalin injection and perfused transcardially with $200 \mathrm{~mL}$ of $2 \%$ sodium nitrate and $2 \%$ heparin mixed with saline, followed by $500 \mathrm{~mL}$ of $4 \%$ paraformaldehyde. The spinal cord was removed via dorsal laminectomy, and the L6$\mathrm{S} 1$ segments were retrieved. The segments were placed in $4 \%$ paraformaldehyde at $4^{\circ} \mathrm{C}$ for 1 day, followed by $30 \%$ buffered sucrose. Using a freezing microtome (Microm, Walldorf, Germany), the spinal cord was cut into $40-\mu \mathrm{m}$ sections.

\section{Functional evaluation}

Cystometrograms were performed on unrestrained conscious rats (Fig. 1). After the onset of general anesthesia, the urinary bladder was exposed using a midline abdominal incision. A polyethylene catheter (PE-50) was inserted through a small incision in the bladder dome, followed by purse-string closure of the bladder. The intravesical catheter was connected via a 3-way stopcock to a pressure transducer and syringe pump attached to a PowerLab system (ADInstruments, Dunedin, New Zealand). Intraluminal pressures were recorded in conscious rats during a 2-hour continuous saline infusion $(0.12 \mathrm{~mL} / \mathrm{min})$ to determine cystometric variables. The interval between voiding contractions and nonvoiding contractions, the frequency of nonvoiding contractions, and the amplitude of bladder contractions were calculated. As cystometrography causes mechanical stimulation, which increases c-fos expression, immunohistochemistry was performed at a different time.

\section{Immunohistochemical and immunofluorescent staining}

Every third spinal cord section was washed several times with phosphate-buffered saline. Sections were processed for c-fos immunoreactivity by avidin-biotin complex methods using rabbit anti-Fos (1:1,000, Oncogene, Cambridge, MA, USA). After staining with nickel-enhanced diaminobenzidine, sections were placed on glass slides, which were air-dried, dehydrated, and covered with a coverslip using standard methods.

Slides were processed immunohistochemically with rabbit anti-PRV (1:1,000, Oncogene, Cambridge, MA, USA) at $4^{\circ} \mathrm{C}$ for 18 hours, followed by donkey anti-rabbit conjugated with fluorescein isothiocyanate (FITC) (1:50, Jackson Immunoresearch, West Grove, PA, USA) for 30 minutes. Slides were then mounted on gelatinized slides, cover-slipped with a fade-retardant medium, and examined under epifluorescent illumination.

Double-labeling was performed in a similar manner. For cfos and PRV double-labeling, slides were processed with goat anti-Fos (1:1,000, Oncogene) and rabbit anti-PRV (1:1,000, Oncogene), followed by donkey anti-rabbit conjugated with FITC (1:50, Jackson Immunoresearch) and donkey anti-goat conjugated with tetramethylrhodamine isothiocyanate (TRITC) (1:50, Jackson Immunoresearch). For c-fos and ChAT doublelabeling, slides were processed with rabbit anti-Fos (1:5,000, Oncogene) and goat anti-ChAT (1:1,000, Chemicon, Temecula, CA, USA), followed by donkey anti-rabbit conjugated with FITC (1:50, Jackson Immunoresearch) and donkey anti-goat conjugated with TRITC (1:50, Jackson Immunoresearch).

\section{Evaluation of specimens}

Spinal cord sections were analyzed using a microscope connected to a color video camera. Cells exhibiting c-fos immunoreactivity were counted in 4 spinal cord regions based on the method of Birder and deGroat [12] (Fig. 2D). The regions were the medial dorsal horn, lateral dorsal horn, DCM, and sacral parasympathetic nucleus (SPN) at L6 and S1. The mean number of fos-positive cells per section was determined for each re-
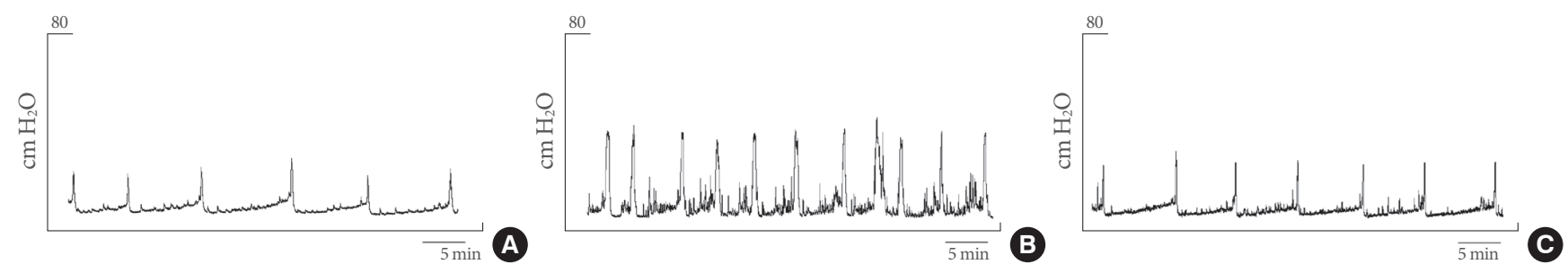

Fig. 1. Cystometrograms of rats in the control (A), prostatitis (B), and capsaicin-pretreated prostatitis groups (C). 

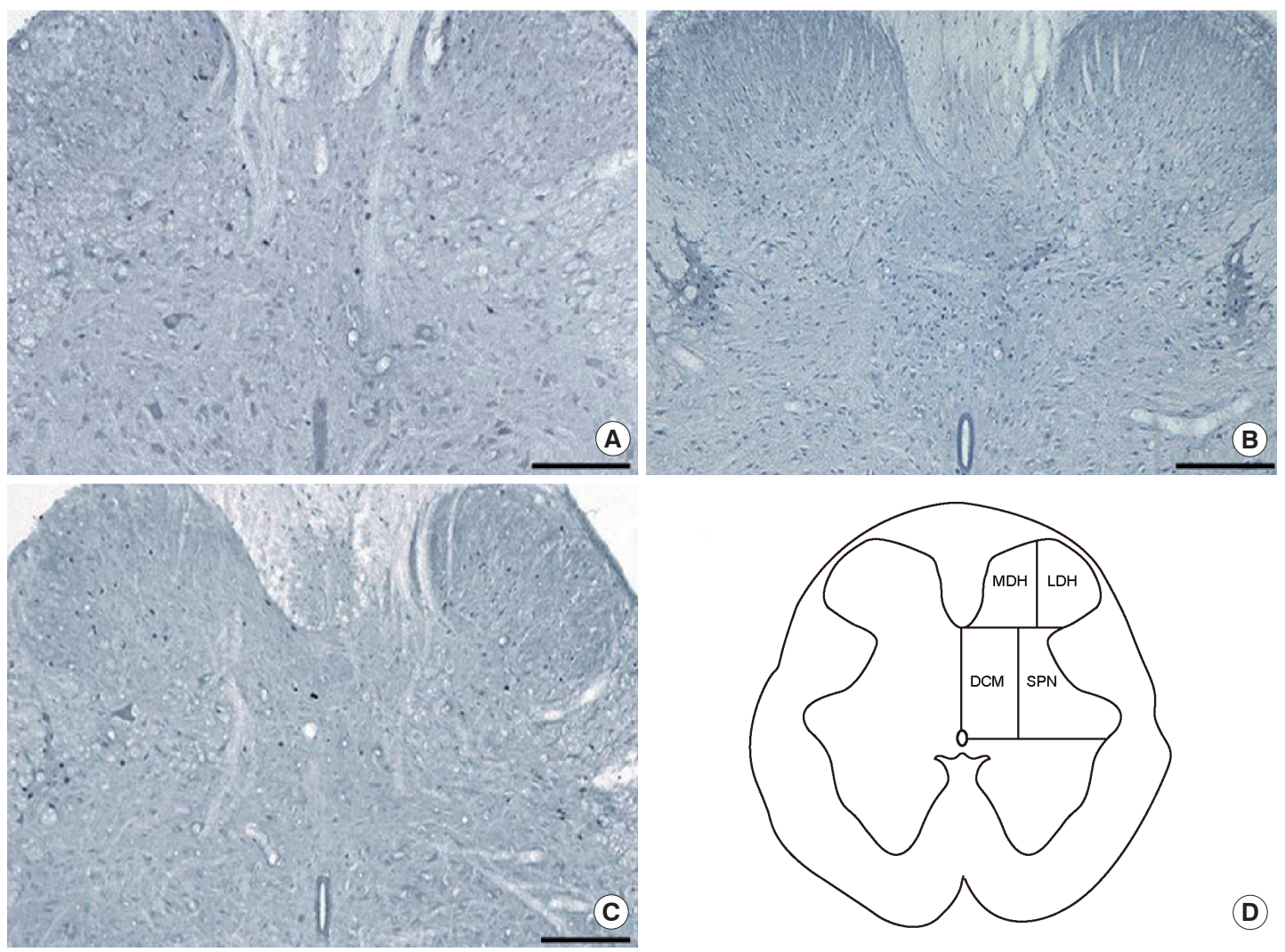

(D)

Fig. 2. Photographs of c-fos-immunoreactive cells in sections of the spinal cord at L6. (A) Normal control group. (B) Prostatitis group. (C) Capsaicin-pretreated prostatitis group. (D) Drawing of a section of the rat spinal cord at L6 depicting the 4 regions where c-fospositive neurons were identified. Calibration bar represents $100 \mu \mathrm{m}$. MDH, medial dorsal horn; $\mathrm{LDH}$, lateral dorsal horn; DCM, dorsal commissure; SPN, sacral parasympathetic nucleus.

gion. Double immunofluorescent staining was performed to evaluate changes in spinal neurons associated with the detrusor muscles.

\section{Statistics}

Data are expressed as mean \pm standard deviation and were evaluated using the t-test, 1-way analysis of variance, and the Tukey multiple comparison test. IBM SPSS Statistics ver. 23.0 (IBM Co., Armonk, NY, USA) was used for statistical analyses. All tests were 2 -tailed. P-values $<0.05$ were considered to indicate statistical significance.

\section{RESULTS}

\section{Functional changes}

After formalin injection into the prostate, the maximal voiding pressure was significantly higher and the intercontraction interval was significantly shorter in the prostatitis group than in the
Table 1. Effects of prostatitis and capsaicin-pretreated prostatitis on cystometrography parameters

\begin{tabular}{lcc}
\hline Group & $\begin{array}{c}\text { Maximal voiding } \\
\text { pressure }\left(\mathrm{cm} \mathrm{H}_{2} \mathrm{O}\right)\end{array}$ & $\begin{array}{c}\text { Intercontraction } \\
\text { interval }(\mathrm{sec})\end{array}$ \\
\hline Control & $25.8 \pm 2.8$ & $275.4 \pm 35.1$ \\
Prostatitis & $37.4 \pm 10.8^{*}$ & $150.6 \pm 14.9^{*}$ \\
Capsaicin-pretreated prostatitis & $29.6 \pm 3.4^{\dagger}$ & $225.7 \pm 28.5$ \\
\hline
\end{tabular}

Values are presented as mean \pm standard deviation.

$\mathrm{P}$-values were obtained using the t-test. ${ }^{*} \mathrm{P}<0.05$ vs. control group. ${ }^{\dagger} \mathrm{P}<0.05$ vs. prostatitis group.

control group (Table 1). There were many nonvoiding detrusor contractions (Fig. 1B). Bladder overactivity was observed in the prostatitis group. The capsaicin-pretreated group had a significantly lower maximal voiding pressure, longer intercontraction interval, and fewer nonvoiding detrusor contractions than the prostatitis group (Fig. 1C). 
Table 2. Number of Fos-immunoreactive cells per a section in different regions of the L6-S1 spinal cord

\begin{tabular}{lccccc}
\hline Group & Total & MDH & LDH & DCM & SPN \\
\hline Control & $10.5 \pm 5.9$ & $1.5 \pm 0.9$ & $1.1 \pm 1.8$ & $2.5 \pm 2.4$ & $5.4 \pm 2.1$ \\
Prostatitis & $80.2 \pm 10.8^{\star}$ & $9.4 \pm 5.6^{*}$ & $12.9 \pm 5.0^{*}$ & $31.8 \pm 7.5^{\star}$ & $25.9 \pm 7.9^{*}$ \\
Capsaicin-pretreated prostatitis & $26.2 \pm 12.4^{\dagger}$ & $3.4 \pm 3.3^{\dagger}$ & $4.4 \pm 3.4^{\dagger}$ & $7.8 \pm 5.2^{\dagger}$ & $10.6 \pm 6.5^{\dagger}$ \\
\hline
\end{tabular}

Values are presented as mean \pm standard deviation.

$\mathrm{MDH}$, medial dorsal horn; LDH, lateral dorsal horn; DCM, dorsal commissure; SPN, sacral parasympathetic nucleus.

P-values were obtained using the t-test. ${ }^{*} \mathrm{P}<0.05$ vs. control group. ${ }^{\dagger} \mathrm{P}<0.05$ vs. prostatitis group.
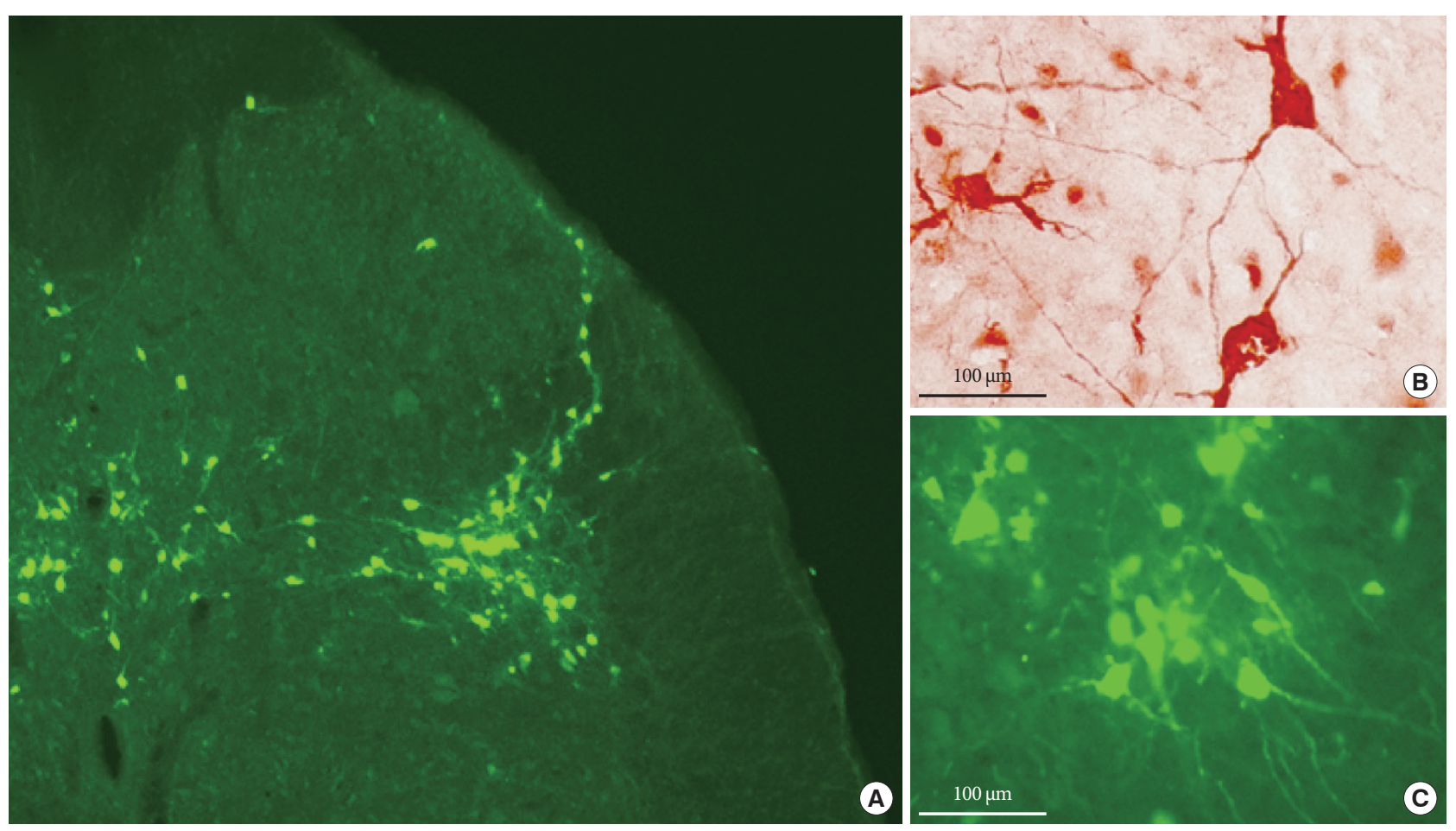

Fig. 3. Pseudorabies virus-immunoreactive (PRV-IR) cells in the spinal cord at L6 after PRV injection into the bladder detrusor muscle. (A) PRV-IR cells in the sacral parasympathetic nucleus and dorsal commissure regions appeared green with immunofluorescent staining for PRV $(\times 40)$. (B) The soma and dendrites of PRV-infected spinal neurons were positively stained after immunochemical staining for PRV. (C) PRV-IR cells appeared green with immunofluorescent staining for PRV at a higher magnification and were similar to the cells identified by immunochemical staining for PRV. Calibration bar represents $100 \mu \mathrm{m}$ in B and C.

\section{Neuronal c-fos expression of the spine}

The number of c-fos-positive cells in the L6 and S1 segments was significantly higher in the prostatitis group $(80.2 \pm 10.8$ cells/section) than in the control group ( $10.5 \pm 5.9$ cells/section) (Fig. 2B, Table 2). The number of c-fos-positive cells was significantly lower in the capsaicin-pretreated prostatitis group (26.2 \pm 12.4 cells/section) than in the prostatitis group (Fig. 2C, Table 2). In all 4 spinal regions, the number of c-fos-positive cells was higher in the prostatitis group than in the control group and significantly lower in the capsaicin-pretreated group than in the prostatitis group (Table 2). Capsaicin-pretreated rats had more c-fos-positive cells than control rats.

\section{PRV immunoreactivity of spinal neurons innervating the bladder}

Three days after PRV infection, a number of PRV-immunoreactive (PVR-IR) cells were observed in the SPN and DCM regions (Fig. 3A). The soma and dendrites of PRV-infected spinal neurons were positive for immunochemical (Fig. 3B) and immunofluorescent (Fig. 3C) staining for PRV. Double PRV and c-fos immunoreactivity was assessed in DCM to determine whether prostatitis activated the interneurons innervating the 

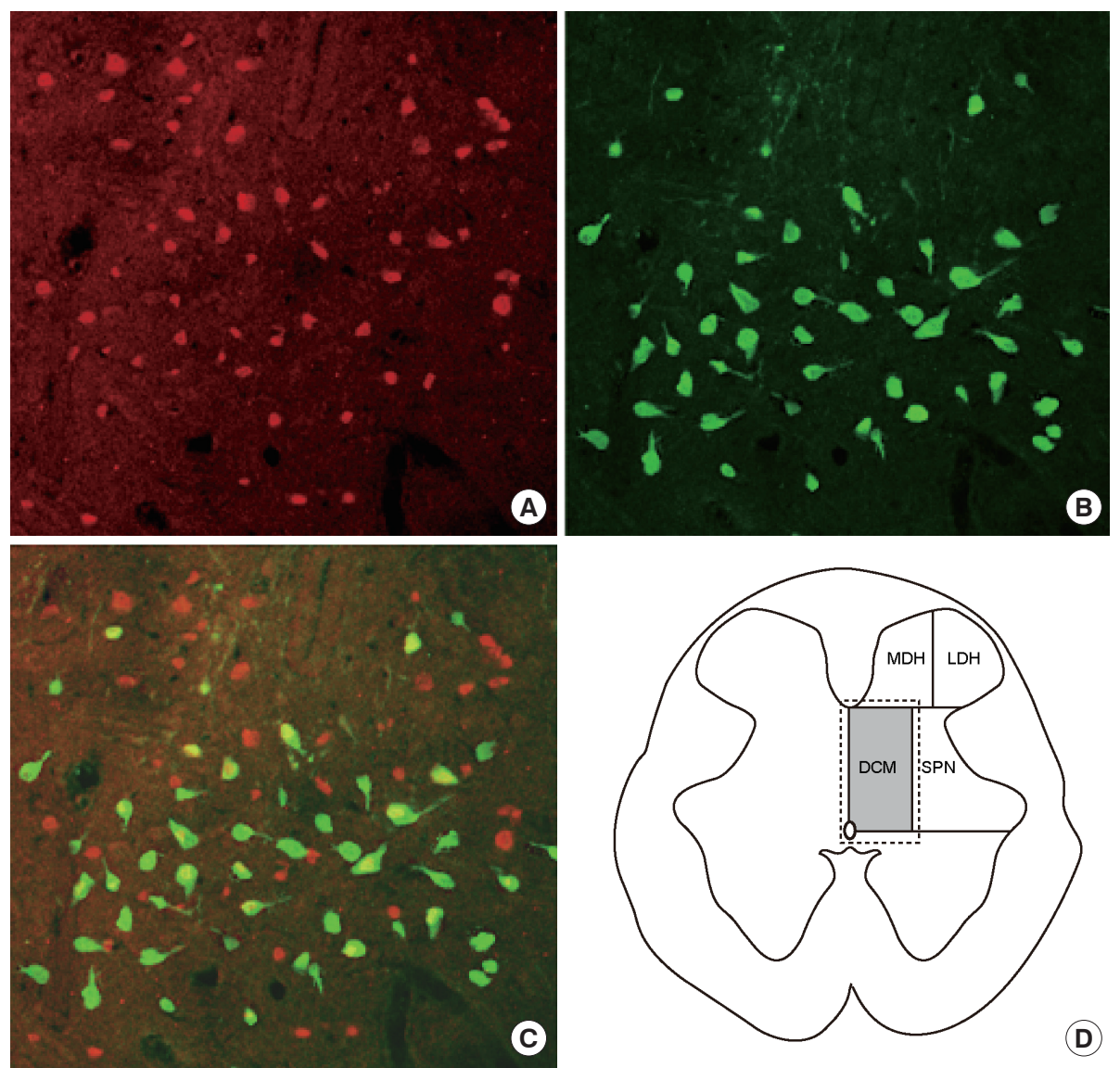

Fig. 4. Pseudorabies virus (PRV) and $c$-fos double immunoreactivity in the dorsal commissure (DCM) region in rats with prostatitis. (A) Fos-immunoreactive (Fos-IR) cells appeared red with immunofluorescent staining for c-fos, and (B) PRV-immunoreactive (PRV-IR) cells appeared green with immunofluorescent staining for PRV. (C) Colocalization of c-fos (red) and PRV (green) was observed in DCM; double-stained cells were yellow. $\mathrm{MDH}$, medial dorsal horn; $\mathrm{LDH}$, lateral dorsal horn; SPN, sacral parasympathetic nucleus.
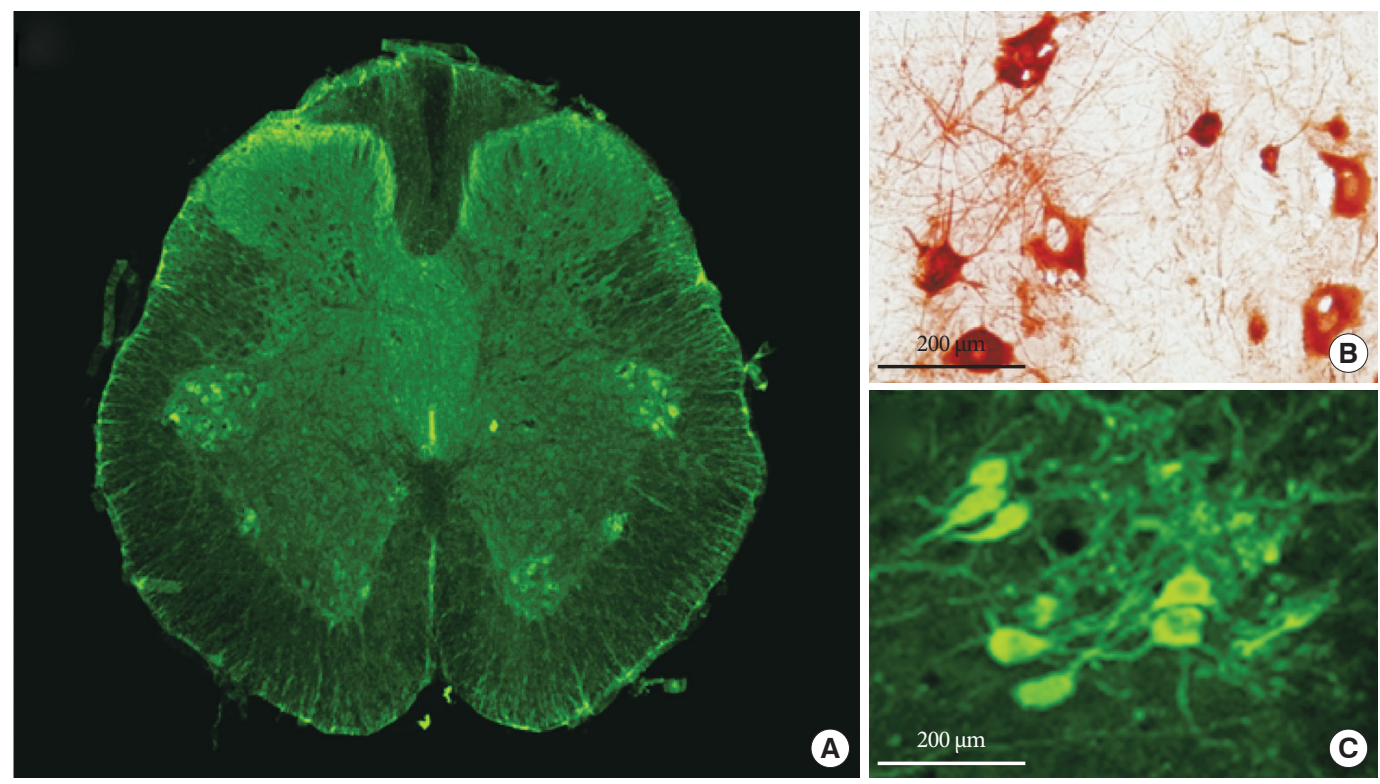

Fig. 5. Choline acetyltransferase-immunoreactive (ChAT-IR) cells in the sacral parasympathetic nucleus (SPN) region of the spinal cord at L6. (A) ChAT-IR cells in SPN appeared green with immunofluorescent staining $(\times 40)$. (B) The soma of preganglionic neurons was positively stained after immunochemical staining for ChAT. (C) ChAT-IR cells appeared green with immunofluorescent staining for ChAT at a higher magnification and were similar to the cells identified by immunochemical staining for ChAT. Calibration bar represents $100 \mu \mathrm{m}$ in B and C. 

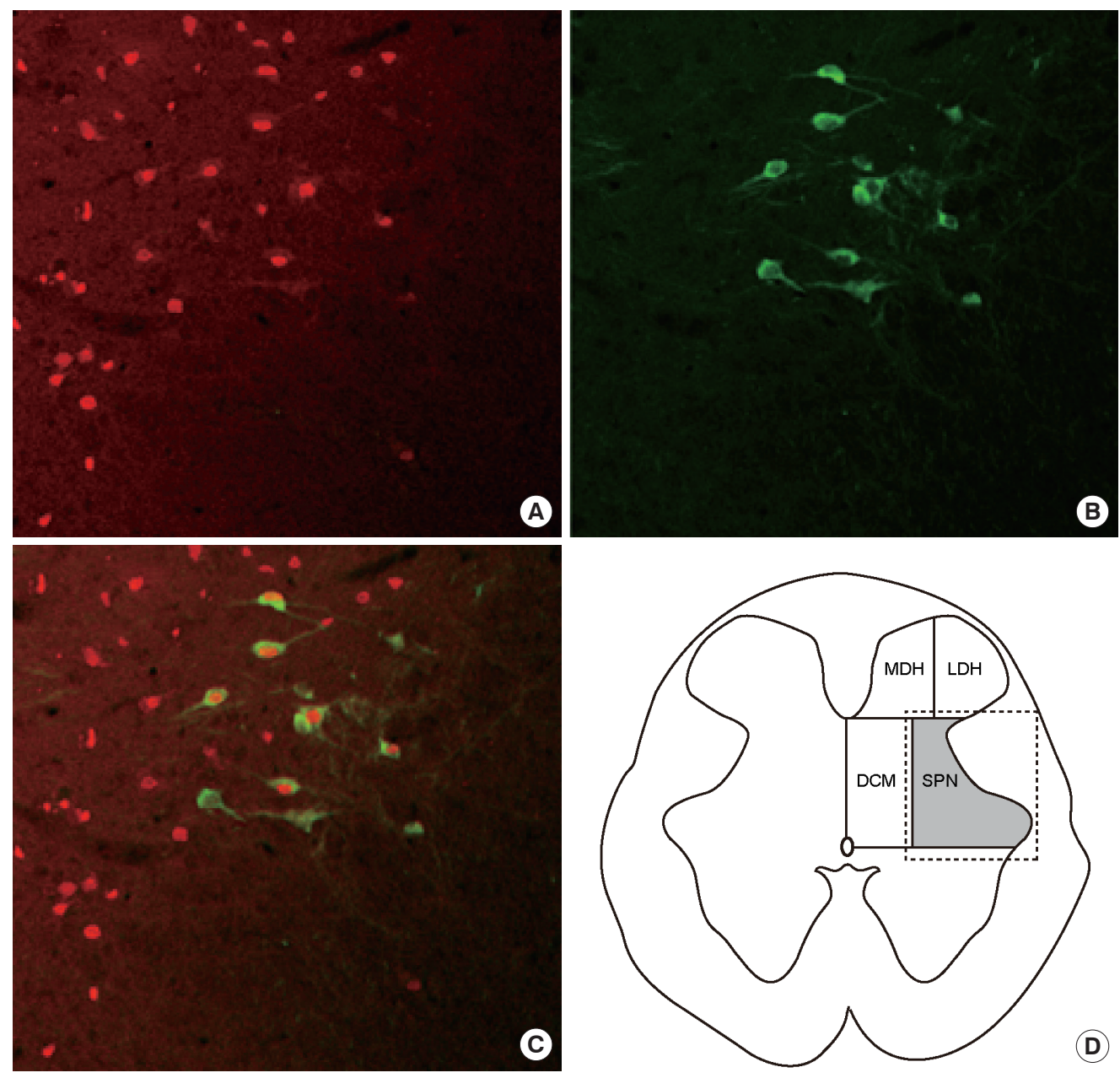

Fig. 6. Choline acetyltransferase (ChAT) and c-fos double immunoreactivity in the sacral parasympathetic nucleus (SPN) region in rats with prostatitis. (A) Fos-immunoreactive (Fos-IR) cells appeared red in the nucleolus of the neurons during immunofluorescent staining for c-fos, and (B) ChAT-IR cells appeared green in the soma of preganglionic neurons during immunofluorescent staining for ChAT. (C) Colocalization of c-fos (red) and ChAT (green) was observed in SPN; double-stained cells were yellow. MDH, medial dorsal horn; LDH, lateral dorsal horn; DCM, dorsal commissure.

bladder. Fos-immunoreactive (Fos-IR) cells were stained red (Fig. 4A) and PRV-IR cells, representing neurons specifically innervating the bladder, were stained green (Fig. 4B). Among the interneurons innervating the bladder, those activated by prostatitis were double-stained, appearing yellow (Fig. 4C).

\section{PGNs in the SPN}

ChAT immunofluorescent staining revealed a number of ChATimmunoreactive (ChAT-IR) neurons (representing PGNs) in the SPN. ChAT staining was noted in a mean of 11.5 PGNs/section (Fig. 5A). The soma of the PGNs showed positive results during immunochemical staining for ChAT (Fig. 5B), and similar findings were noted for immunofluorescent staining (Fig.
5C). To determine whether prostatitis activated bladder PGNs, we evaluated double immunoreactivity with ChAT and c-fos in the SPN region. Fos-IR cells were observed in all SPN areas (Fig. 6A), and ChAT-IR cells were observed in the ventral portion of the SPN (Fig. 6B). Among the PGNs (ChAT-IR cells), 57.4\% were Fos-IR (Fig. 6C).

\section{DISCUSSION}

In current study, we demonstrated that prostatitis induced changes in bladder function through alterations in spinal neuronal activity associated with both the bladder and the prostate. Prostatitis increased the maximal voiding pressure, reduced the 
intercontraction interval, and induced c-fos expression in the lumbosacral cord through a capsaicin-sensitive parasympathetic afferent pathway. The activated spinal neurons included both interneurons and PGNs of the bladder. Using methylene dye mixed with formalin, we confirmed that formalin did not enter the bladder, and the functional changes of the bladder, in our rats with chemical prostatitis, were not caused by direct transmission of inflammation from the prostate to the bladder.

Formalin injection in the prostate significantly increased the quantity of c-fos-positive cells in the L6 and S1 spinal segments, compared with saline injection. This indicates that the lumbosacral spinal cord received nociceptive afferent input from the prostate. Birder and deGroat [12] found that very few c-fospositive cells in the rostral lumbar segments received afferent input via sympathetic nerves during irritation of the bladder by acetic acid or of the prostate by formalin. After chemical-induced prostatitis, most c-fos-positive cells were noted in the DCM (39.8\%); a similar percentage was observed with saline injection (42.2\%). These findings are consistent with those of Birder and deGroat [12], who reported that $48 \%$ of the c-fospositive cells observed after acetic acid-induced bladder irritation were located in the DCM. The DCM, therefore, appears to be the most important region for processing nociceptive input.

The capsaicin-pretreated prostatitis group showed significantly fewer c-fos-positive cells than the prostatitis group. This suggests that capsaicin-sensitive prostate afferents are involved in c-fos expression in the lumbosacral spinal cord after noxious prostatic stimuli. However, despite systemic C-fiber desensitization by subcutaneous capsaicin injections, the number of $\mathrm{c}$-fospositive cells and maximal voiding pressure did not return to the level of the control group, suggesting that capsaicin could not block all C-fibers. Szallasi et al. reported that a single systemic capsaicin injection ( $>50 \mathrm{mg} / \mathrm{kg}$ subcutaneously) could disrupt up to $90 \%$ of A- $\delta$ and C-fibers [13]. Nevertheless, we found that capsaicin pretreatment reduced c-fos-positive cells to almost $25 \%$ of the number without pretreatment, indicating that the main pathway involves C-fibers.

Although our prostatitis group exhibited increased c-fos expression in spinal neurons innervating the bladder, it is uncertain whether activation of prostate afferents by prostatitis caused the functional bladder changes. Spinal neurons associated with sensory neurons are classified as PGNs that send axons to the peripheral nervous system, interneurons that make synaptic contacts with PGNs, and spinal tract neurons that project to the brainstem and diencephalon [14,15]. All 3 types express c-fos after the activation of sensory neurons [14]. Moreover, c-fos-positive cells include neurons from different organs, so it is difficult to distinguish the neuron source by c-fos staining alone. This is a limitation of c-fos staining for observing interactions between organs.

$\mathrm{PRV}$ is a neurotropic virus transported in a retrograde fashion through efferent neuron fibers to the cell bodies of origin [15]. In contrast, anterograde transport of PRV through afferent neuron fibers is slow and incomplete; it is therefore unlikely that interneuron infection in the spine occurs through afferent pathways [16]. Our failure to detect PRV in the dorsal root ganglia or central projection neurons supports this. PRV injected into the bladder first infects PGNs of the lumbosacral spinal cord, followed by the interneurons in direct contact with the PGNs [17]. Immunofluorescent staining with ChAT distinguishes interneurons from PGNs, facilitating the morphological evaluation of interneurons [10]. ChAT staining has also been used to reveal coordinated neuronal pathways of different organs, since it can distinguish neurons innervating specific organs [10].

By simultaneous labeling with 2 immunohistochemically distinguishable forms of PRV, Nadelhaft and Vera [11] identified spinal neuron coordination in the micturition reflex pathway involving the bladder and external urethral sphincter. Although transneuronal tracing studies using PRV allow an assessment of neuron morphology, their usefulness in micturition reflex studies is limited, as they do not allow correlation with sensory neuron activation. In another study using similar labeling methodology, Nadelhaft et al. [10] identified interactions between neurons in the urinary bladder and prostate. However, the immunohistochemically-positive cells were involved in the efferent pathway, and therefore provided no information regarding sensory neurons activated by prostatitis.

To clarify voiding function changes in prostatitis, it is necessary to create an experimental model showing interactions between neurons innervating the prostate and bladder; no clear models have been heretofore established. In this study, we used double immunofluorescent staining for c-fos and PRV, with cfos expression signifying activation of prostate sensory neurons and PRV expression identifying neurons innervating specific organs. The observation of spinal neurons stained positively for both c-fos and PRV revealed the neuronal pathway involved in the prostatitis-induced activation of sensory neurons, which led to the activation of interneurons and PGNs distributed in the bladder and eventual changes in bladder function. This pelvic viscerovisceral reflex can be induced by neurogenic inflammation in the bladder. 
In summary, our results suggest that bladder function changes associated with prostatitis occur via a viscerovisceral reflex pathway, which is activated by sensory neurons in the prostate. These prostate afferents stimulate interneurons and PGNs involved in micturition. Moreover, capsaicin-sensitive fibers are associated with activation of prostate afferents. Our findings provide a theoretical basis for expanding prostatitis treatment beyond simply treating the cause - mainly bacterial infection - to include sensory nerve blockade, based on the role of neurological mechanisms in the pathogenesis of prostatitis symptoms. This treatment expansion correlates with recent trends of using neuromodulation to treat voiding dysfunction, focusing on modulation of sensory neurons [18]. This study not only improves our understanding of the neurological basis of the pathogenesis of prostatitis symptoms, but it may also lead to new treatment modalities that focus on blocking prostate afferents.

\section{AUTHOR CONTRIBUTION STATEMENT}

- Full access to all the data in the study and takes responsibility for the integrity of the data and the accuracy of the data analysis: Jee Soo Park, Mei Hua Jin, Chang Hee Hong

- Study concept and design: Chang Hee Hong

- Acquisition of data: Mei Hua Jin

- Analysis and interpretation of data: Jee Soo Park

- Drafting of the manuscript: Jee Soo Park

-Critical revision of the manuscript for important intellectual content: Chang Hee Hong

-Statistical analysis: Jee Soo Park, Mei Hua Jin

- Obtained funding: Chang Hee Hong

- Administrative, technical, or material support: Chang Hee Hong

- Study supervision: Chang Hee Hong

\section{REFERENCES}

1. Krieger JN, Ross SO, Riley DE. Chronic prostatitis: epidemiology and role of infection. Urology 2002;60(6 Suppl):8-12.

2. Krieger JN, Nyberg L Jr, Nickel JC. NIH consensus definition and classification of prostatitis. JAMA 1999;282:236-7.

3. Pontari MA, Ruggieri MR. Mechanisms in prostatitis/chronic pelvic pain syndrome. J Urol 2004;172:839-45.

4. Rees J, Abrahams M, Doble A, Cooper A; Prostatitis Expert Reference Group (PERG). Diagnosis and treatment of chronic bacterial prostatitis and chronic prostatitis/chronic pelvic pain syndrome: a consensus guideline. BJU Int 2015;116:509-25.
5. Yoshimura N, de Groat WC. Plasticity of Na+ channels in afferent neurons innervating rat urinary bladder following spinal cord injury. J Physiol 1997;503(Pt 2):269-76.

6. Steers WD, Ciambotti J, Etzel B, Erdman S, de Groat WC. Alterations in afferent pathways from the urinary bladder of the rat in response to partial urethral obstruction. J Comp Neurol 1991;310:401-10.

7. Dmitrieva N, McMahon SB. Sensitisation of visceral afferents by nerve growth factor in the adult rat. Pain 1996;66:87-97.

8. Turini D, Beneforti P, Spinelli M, Malagutti S, Lazzeri M. Heat/burning sensation induced by topical application of capsaicin on perineal cutaneous area: new approach in diagnosis and treatment of chronic prostatitis/chronic pelvic pain syndrome? Urology 2006;67:910-3.

9. Vera PL, Meyer-Siegler KL. Inflammation of the rat prostate evokes release of macrophage migration inhibitory factor in the bladder: evidence for a viscerovisceral reflex. J Urol 2004;172 (6 Pt 1):2440-5.

10. Nadelhaft I, Miranda-Sousa AJ, Vera PL. Separate urinary bladder and prostate neurons in the central nervous system of the rat: simultaneous labeling with two immunohistochemically distinguishable pseudorabies viruses. BMC Neurosci 2002;3:8.

11. Nadelhaft I, Vera PL. Separate urinary bladder and external urethral sphincter neurons in the central nervous system of the rat: simultaneous labeling with two immunohistochemically distinguishable pseudorabies viruses. Brain Res 2001;903:33-44.

12. Birder LA, de Groat WC. Increased c-fos expression in spinal neurons after irritation of the lower urinary tract in the rat. J Neurosci 1992;12:4878-89.

13. Szallasi A, Blumberg PM. Vanilloid (Capsaicin) receptors and mechanisms. Pharmacol Rev 1999;51:159-212.

14. Birder LA, Roppolo JR, Erickson VL, de Groat WC. Increased c-fos expression in spinal lumbosacral projection neurons and preganglionic neurons after irritation of the lower urinary tract in the rat. Brain Res 1999;834:55-65.

15. Hamilton MO, Papka RE, O'Donoghue DL, Vaidya AM, Williams SJ, Poff CR, et al. Spinal projection neurons to the laterodorsal pontine tegmental nucleus: relationship to preganglionic neurons and nitric oxide synthase. J Comp Neurol 1995;353:1-8.

16. de Groat WC, Kruse MN, Vizzard MA, Cheng CL, Araki I, Yoshimura N. Modification of urinary bladder function after spinal cord injury. Adv Neurol 1997;72:347-64.

17. de Groat WC, Araki I, Vizzard MA, Yoshiyama M, Yoshimura N, Sugaya K, et al. Developmental and injury induced plasticity in the micturition reflex pathway. Behav Brain Res 1998;92:127-40.

18. Wang Y, Hassouna MM. Neuromodulation reduces c-fos gene expression in spinalized rats: a double-blind randomized study. J Urol 2000;163:1966-70. 